\title{
TREEWIDTH OF CARTESIAN PRODUCTS OF HIGHLY CONNECTED GRAPHS
}

\author{
DAVID R. WOOD
}

\begin{abstract}
The following theorem is proved: For all $k$-connected graphs $G$ and $H$ each with at least $n$ vertices, the treewidth of the cartesian product of $G$ and $H$ is at least $k(n-2 k+2)-1$. For $n \gg k$ this lower bound is asymptotically tight for particular graphs $G$ and $H$. This theorem generalises a well known result about the treewidth of planar grid graphs.
\end{abstract}

Treewidth is a graph parameter of fundamental importance in graph minor theory, with numerous applications in algorithmic theory and practical computing. The planar grid graph is a key example for treewidth, in that the $n \times n$ planar grid has treewidth $n$, and every graph with sufficiently large treewidth contains the $n \times n$ planar grid as a minor.

Motivated by the fact that the planar grid can be defined to be the cartesian product of two paths, in this note we consider the treewidth of cartesian products of general graphs. Our main result is a lower bound on the treewidth of the cartesian product of two highly connected graphs; see $[1,5-12,14,15]$ for related results. Before stating the theorem, we introduce the necessary definitions.

The cartesian product of graphs $G$ and $H$, denoted by $G \square H$, is the graph with vertex set $V(G \square H):=V(G) \times V(H)$, where $(v, x)(w, y)$ is an edge of $G \square H$ if and only if $v w \in E(G)$ and $x=y$, or $v=w$ and $x y \in E(H)$. For each vertex $v \in V(G)$ the subgraph of $G \square H$ induced by $\{(v, w): w \in V(H)\}$ is isomorphic to $H$; we call it the $v$-copy of $H$, denoted by $H_{v}$. Similarly, for each vertex $w \in V(H)$ the subgraph of $G \square H$ induced by $\{(v, w): v \in V(G)\}$ is isomorphic to $G$; we call it the $w$-copy of $G$, denoted by $G_{w}$.

A tree decomposition of a graph $G$ consists of a tree $T$ and a set $\left\{T_{x} \subseteq V(G): x \in\right.$ $V(T)\}$ of 'bags' of vertices of $G$ indexed by $T$, such that

- for each edge $v w \in E(G)$, some bag $T_{x}$ contains both $v$ and $w$, and

- for each vertex $v \in V(G)$, the set $\left\{x \in V(T): v \in T_{x}\right\}$ induces a non-empty (connected) subtree of $T$.

Date: March 25, 2022.

Department of Mathematics and Statistics, The University of Melbourne, Australia (woodd@unimelb.edu.au). Supported by QEII Research Fellowship from the Australian Research Council. 
The width of the tree decomposition is $\max \left\{\left|T_{x}\right|: x \in V(T)\right\}-1$. The treewidth of $G$, denoted by $\operatorname{tw}(G)$, is the minimum width of a tree decomposition of $G$. For example, $G$ has treewidth 1 if and only if $G$ is a forest.

Let $G$ be a graph. Two subgraphs $X$ and $Y$ of $G$ touch if $X \cap Y \neq \emptyset$ or there is an edge of $G$ between $X$ and $Y$. A bramble in $G$ is a set of pairwise touching connected subgraphs. A set $S$ of vertices in $G$ is a hitting set of a bramble $\mathcal{B}$ if $S$ intersects every element of $\mathcal{B}$. The order of $\mathcal{B}$ is the minimum size of a hitting set. The canonical example of a bramble of order $\ell$ is the set of crosses (union of a row and column) in the $\ell \times \ell$ grid. The following 'Treewidth Duality Theorem' shows the intimate relationship between treewidth and brambles; see [2] for an alternative proof.

Theorem 1 ([13]). A graph $G$ has treewidth at least $\ell$ if and only if $G$ contains a bramble of order at least $\ell+1$.

This paper proves the following general lower bound on the treewidth of cartesian products of highly connected graphs.

Theorem 2. For all $k$-connected graphs $G$ and $H$ each with at least $n$ vertices,

$$
\operatorname{tw}(G \square H) \geq k(n-2 k+2)-1 .
$$

Proof. To prove this theorem, we construct a bramble $\mathcal{B}$ in $G \square H$ and then apply Theorem 1 . If $n \leq 2 k-2$ then the claim is vacuously true. Now assume that $n \geq 2 k-1$.

Let $\mathcal{B}$ be the set of all subgraphs $X$ of $G \square H$ formed in the following way. Let $S$ be a set of $2 k-1$ vertices in $G$. Let $T$ be a set of $2 k-1$ vertices in $H$. Initialise $X$ to be the union of $\cup\left\{H_{v}: v \in S\right\}$ and $\cup\left\{G_{w}: w \in T\right\}$. Now delete vertices from $X$ such that at most $k-1$ vertices are deleted from $H_{v}$ for each $v \in S$, and at most $k-1$ vertices are deleted from $G_{w}$ for each $w \in T$. We claim that $\mathcal{B}$ is a bramble of $G \square H$.

First we prove that each $X \in \mathcal{B}$ is connected. Say $X$ is defined with respect to $S \subseteq V(G)$ and $T \subseteq V(H)$. First note that for each $v \in S$ and $w \in T$, since $H_{v}$ and $G_{w}$ are $k$-connected, $H_{v} \cap X$ and $G_{w} \cap X$ are connected. Let $Q$ be the bipartite graph with $V(Q)=S \cup T$, where for all $v \in S$ and $w \in T$, the edge $v w$ is in $Q$ whenever the vertex $(v, w)$ is in $X$ (i.e., it was not deleted). The degree in $Q$ of each vertex $v \in S$ is at least $(2 k-1)-(k-1)=k$ since at most $k-1$ vertices were deleted from $H_{v}$. Similarly, each vertex in $T$ has degree at least $k$ in $Q$. So $Q$ has $2 k-1$ vertices in each colour class, and minimum degree $k$. If $Q$ is disconnected then some component $H$ of $Q$ contains at most $k-1$ vertices in $S$, implying that the vertices in $H \cap T$ have degree at most $k-1$. Thus $Q$ is connected. Now consider two vertices $\left(v_{1}, w_{1}\right)$ and $\left(v_{2}, w_{2}\right)$ in $X$. Thus $v_{1} w_{1}$ and $v_{2} w_{2}$ are edges of $Q$. Since $Q$ is connected, there is a path $P$ in $Q$ between one endpoint of $v_{1} w_{1}$ and one endpoint of $v_{2} w_{2}$. For each 2-edge path $v w v^{\prime}$ of $P$, since $G_{w} \cap X$ is connected, there is a path in $X$ between the vertices $(v, w)$ and $\left(v^{\prime}, w\right)$. Similarly, for 
each 2-edge path $w v w^{\prime}$ of $P$, there is a path in $X$ between the vertices $(v, w)$ and $\left(v, w^{\prime}\right)$. The union of these paths is a walk between $\left(v_{1}, w_{1}\right)$ and $\left(v_{2}, w_{2}\right)$ in $X$. Therefore $X$ is connected, as claimed.

Now we prove that $X$ and $X^{\prime}$ touch for all $X, X^{\prime} \in \mathcal{B}$. Say $X$ is defined with respect to $S$ and $T$, and $X^{\prime}$ is defined with respect to $S^{\prime}$ and $T^{\prime}$. At most $(2 k-1)(k-1)$ vertices in $S \times T^{\prime}$ were deleted in the construction of $X$, and at most $(2 k-1)(k-1)$ vertices in $S \times T^{\prime}$ were deleted in the construction of $X^{\prime}$. Since $\left|S \times T^{\prime}\right|=(2 k-1)^{2}>2(2 k-1)(k-1)$, some vertex $(v, w) \in S \times T^{\prime}$ was deleted in neither the construction of $X$ nor the construction of $X^{\prime}$. Hence $(v, w)$ is in both $H_{v} \cap X$ and $G_{w} \cap X$. Thus $X$ and $X^{\prime}$ have a common vertex.

Therefore $\mathcal{B}$ is a bramble. Let $J$ be a hitting set of $\mathcal{B}$. We claim that $|J| \geq k(n-2 k+2)$. Let $S_{0}:=\left\{v \in V(G):\left|V\left(H_{v}\right) \cap J\right| \leq k-1\right\}$ and $T_{0}:=\left\{w \in V(H):\left|V\left(G_{w}\right) \cap J\right| \leq k-1\right\}$. If $\left|S_{0}\right| \leq 2 k-2$ then at least $n-(2 k-2)$ pairwise-disjoint copies of $H$ contain at least $k$ vertices in $J$, implying $|J| \geq k(n-2 k+2)$, as claimed. Otherwise, $\left|S_{0}\right| \geq 2 k-1$. Similarly, $\left|T_{0}\right| \geq 2 k-1$. Let $S \subseteq S_{0}$ and $T \subseteq T_{0}$ such that $|S|=|T|=2 k-1$. Let $X$ be the union of $\cup\left\{H_{v}-J: v \in S\right\}$ and $\cup\left\{G_{w}-J: w \in T\right\}$. Thus $X \in \mathcal{B}$ (since $\left|V\left(H_{v} \cap J\right)\right| \leq k-1$ and $\left|V\left(G_{w} \cap J\right)\right| \leq k-1$ for each $v \in S$ and $\left.w \in T\right)$. However, $X \cap J=\emptyset$. Thus $J$ is not a hitting set for $\mathcal{B}$. Hence the order of $\mathcal{B}$ is at least $k(n-2 k+2)$. The result follows from Theorem 1.

We now show that the bound in Theorem 2 is tight (ignoring lower order terms and assuming $n \gg k$ ). The bandwidth of a graph $G$, denoted by $\operatorname{bw}(G)$, is the minimum, taken over all bijections $\phi: V(G) \rightarrow\{1,2, \ldots,|V(G)|\}$, of the maximum, taken over all edges $v w \in E(G)$, of $|\phi(v)-| \phi(w) \mid$. It is well known that $\operatorname{tw}(G) \leq \operatorname{bw}(G)$; see [3]. Let $P_{n}^{k}$ be the $k$-th power of a path, which has vertex set $\{1,2, \ldots, n\}$, where $i j$ is an edge if and only if $|i-j| \leq k$. Clearly $P_{n}^{k}$ is $k$-connected. Let $\phi$ be the vertex ordering of $P_{n}^{k} \square P_{n}^{k}$ defined by $\phi((x, y))=(x-1) n+y$. Each edge $(x, y)\left(x, y^{\prime}\right)$ has width $\left|y^{\prime}-y\right| \leq k$, and each edge $(x, y)\left(x^{\prime}, y\right)$ has width $\left|\left(x^{\prime}-x\right) n\right| \leq k n$. Hence $\operatorname{tw}\left(P_{n}^{k} \square P_{n}^{k}\right) \leq \operatorname{bw}\left(P_{n}^{k} \square P_{n}^{k}\right) \leq k n$. (This upper bound can be slightly improved by ordering the vertices with respect to the function $x(n+1)+y n$.)

In fact, there is a much broader class of graphs that provide an upper bound only slightly weaker than $k n$. Let $G$ and $H$ be chordal graphs with $n$ vertices and connectivity $k$. It is well known that $G$ and $H$ have clique-number $k+1$ and treewidth $k$. A tree decomposition of $G$ with width $k$ can be easily turned into a tree decomposition of $G \square H$ with width $(k+1) n-1$; see $[7,14]$. Thus $\operatorname{tw}(G \square H) \leq(k+1) n-1$.

We expect that the dependence on $k$ in Theorem 2 can be slightly improved (although it is not obvious how to do so). For example, Theorem 2 with $k=1$ implies that the $n \times n$ grid has treewidth at least $n-1$, whereas it actually has treewidth $n$; see [4] for 
a proof. Another interesting example is the toroidal grid graph $C_{n} \square C_{n}$. By Theorem 2 with $k=2$ and since $C_{n}$ is a subgraph of $P_{n}^{2}$,

$$
2 n-5 \leq \operatorname{tw}\left(C_{n} \square C_{n}\right) \leq \operatorname{tw}\left(P_{n}^{2} \square P_{n}^{2}\right) \leq 2 n
$$

\section{REFERENCES}

[1] József Balogh, Sergei L. Bezrukov, Lawrence H. Harper, and Ákos Seress. On the bandwidth of 3-dimensional Hamming graphs. Theoret. Comput. Sci., 407(1-3):488-495, 2008. doi:10.1016/j.tcs.2008.07.029.

[2] Patrick Bellenbaum and Reinhard Diestel. Two short proofs concerning tree-decompositions. Combin. Probab. Comput., 11(6):541-547, 2002. doi:10.1017/S0963548302005369.

[3] Hans L. Bodlaender. A partial $k$-arboretum of graphs with bounded treewidth. Theoret. Comput. Sci., 209(1-2):1-45, 1998. doi:10.1016/S0304-3975(97)00228-4.

[4] Hans L. Bodlaender, Alexander Grigoriev, and Arie M. C. A. Koster. Treewidth lower bounds with brambles. Algorithmica, 51:81-98, 2008. doi:10.1007/s00453-007-9056-z.

[5] L. Sunil Chandran and T. Kavitha. The treewidth and pathwidth of hypercubes. Discrete Math., 306(3):359-365, 2006. doi:10.1016/j.disc.2005.12.010.

[6] Jarmila Chvátalová. Optimal labelling of a product of two paths. Discrete Math., 11:249-253, 1975.

[7] Selma Djelloul. Treewidth and logical definability of graph products. Theoret. Comput. Sci., 410(8-10):696-710, 2009. doi:10.1016/j.tcs.2008.10.019.

[8] Carl H. FitzGerald. Optimal indexing of the vertices of graphs. Math. Comp., 28:825-831, 1974. doi:10.2307/2005704.

[9] LaWrence H. Harper. Optimal numberings and isoperimetric problems on graphs. J. Combinatorial Theory, 1:385-393, 1966.

[10] Lawrence H. Harper. On the bandwidth of a Hamming graph. Theoret. Comput. Sci., 301(1-3):491-498, 2003. doi:10.1016/S0304-3975(03)00052-5.

[11] Toru Kojima And Kiyoshi Ando. Bandwidth of the Cartesian product of two connected graphs. Discrete Math., 252(1-3):227-235, 2002. doi:10.1016/S0012-365X (01)00455-1.

[12] Yota Otachi And Ryohei Suda. Bandwidth and pathwidth of threedimensional grids. Discrete Mathematics, 311(10-11):881 - 887, 2011. doi:DOI: 10.1016/j.disc.2011.02.019. 
[13] Paul D. Seymour and Robin Thomas. Graph searching and a min$\max$ theorem for tree-width. J. Combin. Theory Ser. B, 58(1):22-33, 1993. doi:10.1006/jctb.1993.1027.

[14] David R. Wood. Clique minors in cartesian products of graphs, 2007. http://arxiv.org/abs/0711.1189. Submitted.

[15] ZeFang Wu, Xu Yang, And Qinglin Yu. A note on graph minors and strong products. Appl. Math. Lett., 23(10):1179-1182, 2010. doi:10.1016/j.aml.2010.05.007. 